\title{
ON VECTOR BUNDLES OVER REDUCIBLE CURVES WITH A NODE
}

\author{
FILIPPO F. FAVALE AND SONIA BRIVIO
}

\begin{abstract}
Let $C$ be a curve with two smooth components and a single node. Let $\mathcal{U}_{C}(r, w, \chi)$ be the moduli space of $w$-semistable classes of depth one sheaves on $C$ having rank $r$ on both components and Euler characteristic $\chi$. In this paper, under suitable assumptions, we produce a projective bundle over the product of the moduli spaces of semistable vector bundles of rank $r$ on each components and we show that it is birational to an irreducible component of $\mathcal{U}_{C}(r, w, \chi)$. Then we prove the rationality of the closed subset containing vector bundles with given fixed determinant.
\end{abstract}

\section{INTRODUCTION}

Moduli spaces of vector bundles on curves have always been a central topic in Algebraic Geometry. The construction of moduli space of isomorphism classes of stable vector bundle of rank $r$ and degree $d$ on a smooth projective curve of genus $g \geq 2$ is due to Mumford (see [Mum65]). Such a moduli space is a non-singular quasi-projective variety, whose compactification was obtained by Seshadri in [Ses67], by introducing $S$-equivalence relation between semistable vector bundles, and it is denoted by $\mathcal{U}_{C}(r, d)$. The compactification is a normal irreducible projective variety of dimension $r^{2}(g-1)+1$. When $r$ and $d$ are coprime, the notion of semistability coincides with that of stability, so $\mathcal{U}_{C}(r, d)$ parametrizes isomorphism classes of stable vector bundles. Moreover, in this case there exists a Poincaré bundle on $\mathcal{U}_{C}(r, d)$, see [Ram73]. If $L \in \operatorname{Pic}^{d}(C)$ is a line bundle, the moduli space $\mathcal{S} U_{C}(r, L)$, parametrizing semistable vector bundles of rank $r$ and fixed determinat $L$, is also of great interest. Indeed, up to a finite étale covering, the moduli space $\mathcal{U}_{C}(r, d)$ is isomorphic to the product of $\mathcal{S} U_{C}(r, L)$ and $\operatorname{Pic}^{0}(C)$. Hence, a lot of the geometry of $\mathcal{U}_{C}(r, d)$ is encoded in $\mathcal{S} U_{C}(r, L)$. Moreover, $\mathcal{S} U_{C}(r, L)$ is interesting on its own and it is a rational variety when $r$ and $d$ are coprime, see [KS99]. The geometry of these moduli spaces has been studied by many authors, in particular its relation with generalized theta functions, see [Bea13] for a survey, (see [BF19a], [Bri18], [Bri17], [Bri15], [BB12] and [BV12] for recent works by the authors).

Unfortunately, as soon as the base curve becomes singular, the above results do not apply anymore. For example, for a singular irreducible curve, in order to have a compact moduli space one possible approach consists in considering torsion-free sheaves instead of locally free, see [New78] and [Ses82]. This method was generalized for a reducible (but reduced) curve by Seshadri. Its idea was to include in the moduli space also depth one sheaves and to introduce the notion of polarization $w$ and of $w$ semistability. More precisely, we denote by $\mathcal{U}_{C}(w, r, \chi)$ the moduli space parametrizing $w$-semistable sheaves of depth one of rank $r$ on each components and Euler characteristic $\chi$.

Date: July 29, 2020.

2010 Mathematics Subject Classification: Primary: 14H60; Secondary: 14D20

Keywords: Stability, Vector Bundles, Nodal curves.

Both authors are partially supported by INdAM - GNSAGA. 
In this paper we will assume that $C$ is a nodal reducible curve with two smooth irreducible components $C_{1}$ and $C_{2}$, of genera $g_{i} \geq 1$ and a single node $p$. We can get the curve by gluing $C_{1}$ and $C_{2}$ at the points $q_{1}$ and $q_{2}$. In this hypothesis, the moduli space $\mathcal{U}_{C}(w, r, \chi)$ is a connected reducible projective variety, see [TiB95] and [TiB11]; each irreducible component has dimension $r^{2}\left(p_{a}(C)-1\right)+1$ and it corresponds to a possible pair of multidegree, see Section 2 for details. For problems about the stability of Kernel bundles on such curves the reader can see [BF19b].

In the above hypothesis, for any $r \geq 2$, fix a pair of integers $\left(d_{1}, d_{2}\right)$ which are both coprime with $r$. The existence of Poincaré vector bundles, on the moduli spaces $\mathcal{U}_{C_{i}}\left(r, d_{i}\right)$, allows us to produce a projective bundle $\pi: \mathbb{P}(\mathcal{F}) \rightarrow \mathcal{U}_{C_{1}}\left(r, d_{1}\right) \times \mathcal{U}_{C_{2}}\left(r, d_{2}\right)$, whose fiber at $\left(\left[E_{1}\right],\left[E_{2}\right]\right)$ is $\mathbb{P}\left(\operatorname{Hom}\left(E_{1, q_{1}}, E_{2, q_{2}}\right)\right)$, see Lemma 3.1. Let $\left.u \in \mathbb{P}(\mathcal{F}), u=\left(\left(E_{1}\right],\left[E_{2}\right]\right),[\sigma]\right)$, where $\sigma$ is a non zero homomorphism $E_{1, q_{1}} \rightarrow E_{2, q_{2}}$. We can associate to $u$ a depth one sheaf $E_{u}$ on the curve $C$, which is obtained, roughly speaking, by gluing $E_{1}$ and $E_{2}$ along the fibers at $q_{1}$ and $q_{2}$ with $\sigma$. This is a vector bundle if and only if $\sigma$ is an isomorphism. Our first concern is to study when $E_{u}$ turns out to be $w$-semistable for some polarization $w$ : we are able to give some necessary and sufficient conditions to ensure $w$-semistability (see section 3). Then we turn our attention to the rational map

$$
\phi: \mathbb{P}(\mathcal{F})-->\mathcal{U}_{C}(w, r, \chi)
$$

sending $u$ to $E_{u}$. Our first result (Theorem 4.1) can be summarized in the following statement:

Theorem $\mathbf{A}$ Let $C$ be a reducible nodal curve as above. Let $r \geq 2$ and $d_{1}$ and $d_{2}$ be integer coprime with $r$. Set $\chi_{i}=d_{i}+r\left(1-g_{i}\right)$ and $\chi=\chi_{1}+\chi_{2}-r$. For any pair $\left(\chi_{1}, \chi_{2}\right)$ in a suitable non empty subset of $\mathbb{Z}^{2}$ there exists a polarization $w$ such that $\mathbb{P}(\mathcal{F})$ is birational to the irreducible component of the moduli space $\mathcal{U}_{C}(w, r, \chi)$ corresponding to bidegree $\left(d_{1}, d_{2}\right)$.

The birational map of the statement is the map $\phi$. We prove that it is an injective morphism on the open subset $\mathscr{U} \subset \mathbb{P}(\mathcal{F})$, given by points $u$ where $\sigma$ is an isomorphism. The image $\phi(\mathscr{U})$ is a dense subset of the moduli space and its points are classes of vector bundles whose restriction to each component is stable (see Theorem 4.1).

Moreover, when $g_{i}>r+1$, we can give some more information about the domain of $\phi$ as follows, see Theorem 4.3.

Theorem B Assume that the hypothesis of Theorem A hold. If $g_{i}>r+1$, for any pair $\left(\chi_{1}, \chi_{2}\right)$ in a suitable non empty subset of $\mathbb{Z}^{2}$ there exists a non empty open subset $V_{1} \times V_{2}$ of $\mathcal{U}_{C_{1}}\left(r, d_{1}\right) \times \mathcal{U}_{C_{2}}\left(r, d_{2}\right)$ and a polarization $w$ such that $\left.\phi\right|_{\mathscr{U} \cup \mathscr{V}}$ is a morphism, where we set $\mathscr{V}=\pi^{-1}\left(V_{1} \times V_{2}\right)$.

Then, in analogy with the smooth case, for any $L \in \operatorname{Pic}(C)$ we define the variety $\mathcal{S} U_{C}(w, r, L)$ which is, roughly, the closure in $\mathcal{U}_{C}(w, r, \chi)_{d_{1}, d_{2}}$ of the locus parametrising classes of vector bundles with fixed determinant $L$ where $d_{i}=\operatorname{deg}\left(\left.L\right|_{C_{i}}\right)$. When $r$ and $d_{i}$ are coprime, as in the smooth case, we obtain the following result, see Theorem 5.2:

Theorem C Under the hypothesis of Theorem $A, \mathcal{S} U_{C}(w, r, L)$ is a rational variety.

Recent results concerning rationality of these moduli spaces on reducible curves are obtained in [DS18] and [BDS16] in the case of rank two and in [BB14] for an integral irreducible nodal curve.

The paper is organized as follows. In Section 1 we fix notations about reducible nodal curves. In Section 2 we introduce the notion of depth one sheaves, of polarization and $w$-semistability and we recall general properties on their moduli spaces. In Section 3 we introduce the projective bundle $\mathbb{P}(\mathcal{F})$, 
we define the sheaf $E_{u}$ associated to $u \in \mathbb{P}(\mathcal{F})$ and we study when it is $w$-semistable. In Section 4 we prove Theorems A and B. Finally, in Section 5 we deal with moduli spaces with fixed determinant and we prove Theorem $\mathrm{C}$.

\section{Acknowledgements}

We would like to thank Alessandro Verra for comments on a preliminary version of this paper and the referee for several valuable advices. We are grateful to Prof. P.E. Newstead and Prof. A. Dey for suggesting us some references.

\section{NodAl REDUCIBLE CURVES}

In this paper we will consider nodal reducible complex projective curves with two smooth irreducible components and one single node. Let $C$ be such a curve, we consider a normalization map $\nu: C_{1} \sqcup C_{2} \rightarrow$ $C$, where $C_{i}$ is a smooth irreducible curve of genus $g_{i} \geq 1$. Hence $\nu^{-1}(x)$ is a single point except when $x$ is the node $p$ of $C$, in this case $\nu^{-1}(p)=\left\{q_{1}, q_{2}\right\}$ with $q_{j} \in C_{j}$. Since the restriction $\nu_{\mid C_{i}}$ is an isomorphism we will identify $C_{1}$ and $C_{2}$ with the irreducible components of $C$.

Notice that $C$ can be embedded in a smooth surface $X$, on which $C$ is an effective divisor $C=C_{1}+C_{2}$ with $C_{1} C_{2}=1$. Let $J_{C}=O_{X}(-C)$ and $J_{C_{i}}=O_{X}\left(-C_{i}\right)$ be the ideal sheaves of $C$ and $C_{i}$ respectively in $X$, then we have the inclusion $J_{C} \subset J_{C_{i}}$. We have the following commutative diagram

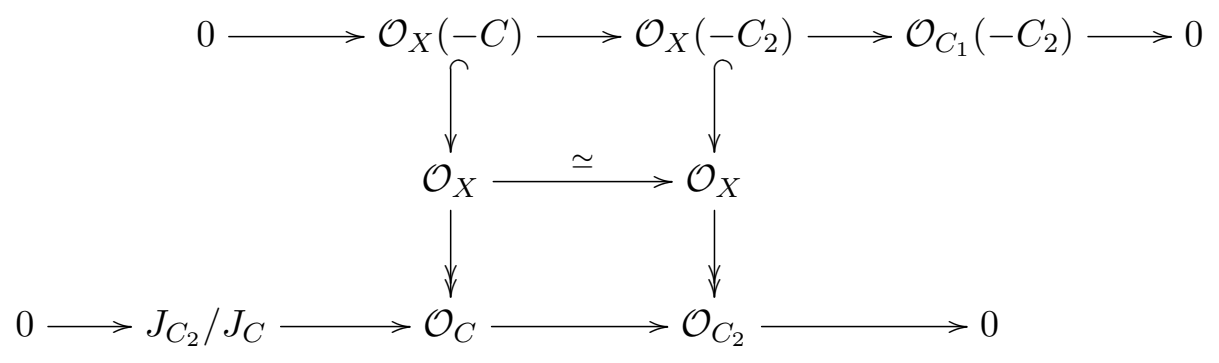

from which one deduces the isomorphism $J_{C_{2}} / J_{C} \simeq O_{C_{1}}\left(-C_{2}\right)$. This gives the exact sequence

$$
0 \rightarrow O_{C_{1}}\left(-C_{2}\right) \rightarrow O_{C} \rightarrow O_{C_{2}} \rightarrow 0 .
$$

which is called the decomposition sequence of $C$. From it we can compute the Euler characteristic of $O_{C}$ :

$$
\chi\left(O_{C}\right)=\chi\left(O_{C_{1}}\left(-C_{2}\right)\right)+\chi\left(O_{C_{2}}\right) .
$$

Let $p_{a}(C)=1-\chi\left(O_{C}\right)$ be the arithmetic genus of $C$, from the above relation we get that $p_{a}(C)=$ $g_{1}+g_{2}$.

Notation 1.1. We will denote by $j_{i}: C_{i} \hookrightarrow C$ the natural inclusion of $C_{i}$ in $C$. We will denote by $\mathcal{O}_{q_{i}}$ the stalk of $\left(j_{i}\right)_{*} \mathcal{O}_{C_{i}}$ in $p$ and by $\mathcal{O}_{p}$ the stalk of $\mathcal{O}_{C}$ in $p$.

\section{Moduli SPACE OF DePth ONE Sheaves}

Let $C$ be a smooth irreducible projective curve of genus $g \geq 1$. The moduli space of semistable vector bundles of rank $r$ and degree $d$ on $C$ will be denoted by $\mathcal{U}_{C}(r, d)$. Its points are $S$-equivalence classes of semistable vector bundles on the curve. We will denote by $[E]$ the class of a vector bundle $E$. In 
[Ses82] it is proved that $\mathcal{U}_{C}(r, d)$ is an irreducible and projective variety. Moreover, see [Ses82], [Tu93], we have:

$$
\operatorname{dim} \mathcal{U}_{C}(r, d)= \begin{cases}r^{2}(g-1)+1 & g \geq 2 \\ \operatorname{gcd}(r, d) & g=1\end{cases}
$$

In particular, when $r$ and $d$ are coprime, $\mathcal{U}_{C}(r, d)$ is a smooth variety, whose points parametrizes isomorphism classes of stable vector bundles. Moreover, for $g=1$, we also have an isomorphism $\mathcal{U}_{C}(r, d) \simeq C$ (see [Ati57] and [Tu93]).

Let $C$ be a nodal curve with a single node $p$ and two smooth irreducible components $C_{1}$ and $C_{2}$. To construct compactifications of moduli spaces of vector bundles on $C$ we introduce depth one sheaves, following the approach of Seshadri, see [Ses82].

Definition 2.1. A coherent sheaf $E$ on $C$ is of depth one if every torsion section vanishes identically on some components of $C$.

A coherent sheaf $E$ on $C$ is of depth one if and only if the stalk at the node $p$ is isomorphic to $\mathcal{O}_{p}^{a} \oplus \mathcal{O}_{q_{1}}^{b} \oplus \mathcal{O}_{q_{2}}^{c}$, see [Ses82]. In particular, any vector bundle $E$ on $C$ is a sheaf of depth one. If $E$ is a sheaf of depth one on $C$, then its restriction $\left.E\right|_{C_{i}}$ is a torsion free sheaf on $C_{i} \backslash p$ (possibly identically zero). Moreover, any subsheaf of $E$ is of depth one too.

Let $E$ be a sheaf of depth one on $C$. We define the relative rank of $E$ on the component $C_{i}$ as the rank of the restriction $E_{i}=E_{\mid C_{i}}$ of $E$ to $C_{i}$

$$
r_{i}=\operatorname{Rk}\left(E_{i}\right)
$$

and the multirank of $E$ as the pair $\left(r_{1}, r_{2}\right)$. We define the relative degree of $E$ with respect to the component $C_{i}$ as the degree of the restriction $E_{i}$

$$
d_{i}=\operatorname{deg}\left(E_{i}\right)=\chi\left(E_{i}\right)-r_{i} \chi\left(O_{C_{i}}\right),
$$

where $\chi\left(E_{i}\right)$ is the Euler characteristic of $E_{i}$. The multidegree of $E$ is the pair $\left(d_{1}, d_{2}\right)$.

Definition 2.2. A polarization $w$ of $C$ is given by a pair of rational weights $\left(w_{1}, w_{2}\right)$ such that $0<w_{i}<1$ and $w_{1}+w_{2}=1$. For any sheaf $E$ of depth one on $C$, of multirank $\left(r_{1}, r_{2}\right)$ and $\chi(E)=\chi$, we define the polarized slope as

$$
\mu_{w}(E)=\frac{\chi}{w_{1} r_{1}+w_{2} r_{2}} .
$$

Definition 2.3. Let $E$ be a sheaf of depth one on $C$. $E$ is said $w$-semistable if for any subsheaf $F \subseteq E$ we have $\mu_{w}(F) \leq \mu_{w}(E)$; $E$ is said w-stable if $\mu_{w}(F)<\mu_{w}(E)$ for all proper subsheaf $F$ of $E$.

For each $w$-semistable sheaf $E$ of depth one on $C$ there exists a finite filtration of sheaves of depth one on $C$ :

$$
0=E^{0} \subset E^{1} \subset E^{2} \subset \cdots \subset E^{k}=E,
$$

such that each quotient $E^{i} / E^{i-1}$ is a $w$-stable sheaf of depth one on $C$ with polarized slope $\mu_{w}\left(E^{i} / E^{i-1}\right)=$ $\mu_{w}(E)$. This is called a Jordan-Holder filtration of $E$. The sheaf

$$
G r_{w}(E)=\oplus_{i=1}^{k} E^{i} / E^{i-1}
$$


is said the graduate sheaf associated to $E$ and it depends only on the isomorphism class of $E$. Let $E$ and $F$ be $w$-semistable sheaves of depth one on $C$. We say that $E$ and $F$ are $S_{w}$-equivalent if and only if $G r_{w}(E) \simeq G r_{w}(F)$. If $E$ and $F$ are $w$-stable sheaves then $S_{w^{-}}$-equivalence is just isomorphism, as in the smooth case.

There exists a moduli space $\mathcal{U}_{C}^{s}\left(w,\left(r_{1}, r_{2}\right), \chi\right)$ parametrizing isomorphism classes of $w$-stable sheaves of depth one on $C$ of multirank $\left(r_{1}, r_{2}\right)$ and given Euler characteristic $\chi$, see [Ses82]. It has a natural compactification $\mathcal{U}_{C}\left(w,\left(r_{1}, r_{2}\right), \chi\right)$, whose points correspond to $S_{w}$-equivalence classes of $w$-semistable sheaves of depth one on $C$ of multirank $\left(r_{1}, r_{2}\right)$ and given Euler characteristic $\chi$. In particular, when $r_{1}=r_{2}=r$, we denote by $\mathcal{U}_{C}(w, r, \chi)$ the corresponding moduli space. In this case we have the following result (see [TiB95] and [TiB11]):

Theorem 2.1. Let $C$ be a nodal curve with a single node $p$ and two smooth irreducible components $C_{i}$ of genus $g_{i} \geq 1, i=1,2$. For a generic polarization $w$ we have the following properties:

(1) any $w$-stable vector bundle $E \in \mathcal{U}_{C}(w, r, \chi)$ satisfies the following condition:

$$
w_{i} \chi(E) \leq \chi\left(E_{i}\right) \leq w_{i} \chi(E)+r
$$

where $E_{i}$ is the restriction of $E$ to $C_{i}$;

(2) if a vector bundle $E$ on $C$ satisfies the above condition for $i=1,2$ and the restrictions $E_{1}$ and $E_{2}$ are semistable vector bundles, then $E$ is w-semistable. Moreover, if at least one of the restrictions is stable, then $E$ is w-stable;

(3) the moduli space $\mathcal{U}_{C}(w, r, \chi)$ is connected, each irreducible component has dimension $r^{2}\left(p_{a}(C)-\right.$ $1)+1$ and it corresponds to the choice of a multidegree $\left(d_{1}, d_{2}\right)$ satisfying conditions 2.4.

Definition 2.4. We denote by $\mathcal{U}_{C}(w, r, \chi)_{d_{1}, d_{2}}$ the irreducible component of $\mathcal{U}_{C}(w, r, \chi)$ corresponding to the multidegree $\left(d_{1}, d_{2}\right)$.

\section{Construction of Depth one sheaves.}

In this section we deal with construction of depth one sheaves on a nodal curve $C$ with two irreducible components and a single node. We begin with the following lemma:

Lemma 3.1. Let $C_{1}$ and $C_{2}$ be smooth complex projective curves of genus $g_{i} \geq 1, i=1,2$, and $q_{i} \in C_{i}$. Fix $r \geq 2$ and $d_{1}, d_{2} \in \mathbb{Z}$ such that $r$ is coprime with both $d_{1}$ and $d_{2}$. Then, there exists a projective bundle

$$
\pi: \mathbb{P}(\mathcal{F}) \rightarrow \mathcal{U}_{C_{1}}\left(r, d_{1}\right) \times \mathcal{U}_{C_{2}}\left(r, d_{2}\right)
$$

such that the fiber over $\left(\left[E_{1}\right],\left[E_{2}\right]\right)$ is $\mathbb{P}\left(\operatorname{Hom}\left(E_{1, q_{1}}, E_{2, q_{2}}\right)\right)$, where $E_{i, q_{i}}$ is the fiber of $E_{i}$ at the point $q_{i}$.

Proof. We recall that, as $r$ and $d_{i}$ are coprime, there exists a Poincaré bundle $\mathcal{P}_{i}$ for the moduli space of semistable vector bundles on $C_{i}$ of rank $r$ and degree $d_{i}$, i.e. a vector bundle $\mathcal{P}_{i}$ on $\mathcal{U}_{C_{i}}\left(r, d_{i}\right) \times C_{i}$ such that $\left.\mathcal{P}_{i}\right|_{\left[E_{i}\right] \times C_{i}} \simeq E_{i}$, under the identification $\left[E_{i}\right] \times C_{i} \simeq C_{i}$. This follows from a result of [Ram73] if $g_{i} \geq 2$ and from the isomorphism $\mathcal{U}_{C_{i}}\left(r, d_{i}\right) \simeq C_{i}$ when $g_{i}=1$.

For $i=1,2$, consider the natural inclusion

$$
\iota_{i}: \mathcal{U}_{C_{i}}\left(r, d_{i}\right) \times q_{i} \hookrightarrow \mathcal{U}_{C_{i}}\left(r, d_{i}\right) \times C_{i},
$$


and the pull back $\iota_{i}{ }^{*}\left(\mathcal{P}_{i}\right)$ of the Poincaré bundle. Since $\mathcal{U}_{C_{i}}\left(r, d_{i}\right) \times q_{i}$ is isomorphic to $\mathcal{U}_{C_{i}}\left(r, d_{i}\right)$, $\iota_{i}{ }^{*}\left(\mathcal{P}_{i}\right)$ can be seen as a vector bundle on $\mathcal{U}_{C_{i}}\left(r, d_{i}\right)$ of rank $r$ whose fiber at $\left[E_{i}\right]$ is actually $E_{i, q_{i}}$.

Note that the product $\mathcal{U}_{C_{1}}\left(r, d_{1}\right) \times \mathcal{U}_{C_{2}}\left(r, d_{2}\right)$ is a smooth irreducible variety. Let $p_{1}$ and $p_{2}$ denote the projections of the product onto factors. We define on $\mathcal{U}_{C_{1}}\left(r, d_{1}\right) \times \mathcal{U}_{C_{2}}\left(r, d_{2}\right)$ the following sheaf:

$$
\mathcal{F}:=\mathcal{H o m}\left(p_{1}^{*}\left(\iota_{1}^{*}\left(\mathcal{P}_{1}\right)\right), p_{2}^{*}\left(\iota_{2}^{*}\left(\mathcal{P}_{2}\right)\right)\right)
$$

By construction, $\mathcal{F}$ is a vector bundle of rank $r^{2}$ whose fiber at the point $\left(\left[E_{1}\right],\left[E_{2}\right]\right)$ is $\operatorname{Hom}\left(E_{1, q_{1}}, E_{2, q_{2}}\right)$. By taking the associated projective bundle we conclude the proof.

Let $C_{1}$ and $C_{2}$ be smooth irreducible curves, we consider a nodal curve $C$ with two smooth components and a single node $p$ which is obtained by identifying the points $q_{1} \in C_{1}$ and $q_{2} \in C_{2}$. Let $E_{i}$ be a stable vector bundle of rank $r$ and degree $d_{i}$ on $C_{i}$ and consider a non zero homomorphism $\sigma: E_{1, q_{1}} \rightarrow E_{2, q_{2}}$ between the fibres. Assume that the rank of $\sigma$ is $k$, with $1 \leq k \leq r$. We can associate to these data a depth one sheaf on the nodal curve $C$, roughly speaking, by gluing the vector bundles $E_{1}$ and $E_{2}$ along the fibers (at $q_{1}$ and $q_{2}$ respectively) with the homomorphism $\sigma$, as follows.

Let $j_{p}$ be the inclusion of $p$ in $C$ and let $j_{i}: C_{i} \rightarrow C$ be the inclusion of $C_{i}$ in $C$, for $i=1,2$. The sheaf $j_{i *} E_{i}$ is a depth one sheaf on $C$ whose stalk at $p$ is the stalk of $E_{i}$ at $q_{i}$. Hence, there is a natural surjective map given by restriction onto the fiber of $E_{i}$ at $q_{i}$, i.e. the map

$$
\rho_{i}: j_{i_{*}} E_{i} \rightarrow E_{i, q_{i}}
$$

The sheaf $j_{1 *}\left(E_{1}\right) \oplus j_{2 *}\left(E_{2}\right)$ is of depth one on $C$ and we have a surjective map

$$
\rho_{1} \oplus \rho_{2}: j_{1 *} E_{1} \oplus j_{2 *} E_{2} \rightarrow E_{1, q_{1}} \oplus E_{2, q_{2}}
$$

The sheaf $j_{p_{*}} j_{p}{ }^{*} j_{2 *}\left(E_{2}\right)$ has depth one too, and it is a skyscraper sheaf over $p$ whose stalk is $E_{2, q_{2}}$. So we have again a surjective map

$$
\rho: j_{p_{*}} j_{p}{ }^{*} j_{2 *}\left(E_{2}\right) \rightarrow E_{2, q_{2}}
$$

Let $\sigma: E_{1, q_{1}} \rightarrow E_{2, q_{2}}$ be a non zero homomorphism and consider the induced surjective map

$$
\sigma \oplus i d: E_{1, q_{1}} \oplus E_{2, q_{2}} \rightarrow \operatorname{Im}(\sigma) \oplus E_{2, q_{2}} .
$$

We have, moreover, the map

$$
\delta: \operatorname{Im}(\sigma) \oplus E_{2, q_{2}} \rightarrow E_{2, q_{2}}
$$

which sends $(u, v)$ to $u-v$. We denote by $\Delta \subset \operatorname{Im}(\sigma) \oplus \operatorname{Im}(\sigma)$ the diagonal. By construction we have $\Delta \simeq \mathbb{C}_{p}^{k}$

Finally we define the map of sheaves

$$
\tilde{\sigma}: j_{1 *}\left(E_{1}\right) \oplus j_{2 *}\left(E_{2}\right) \rightarrow j_{p_{*}} j_{p}{ }^{*} j_{2 *}\left(E_{2}\right)
$$

by requiring that the following diagram commutes. 


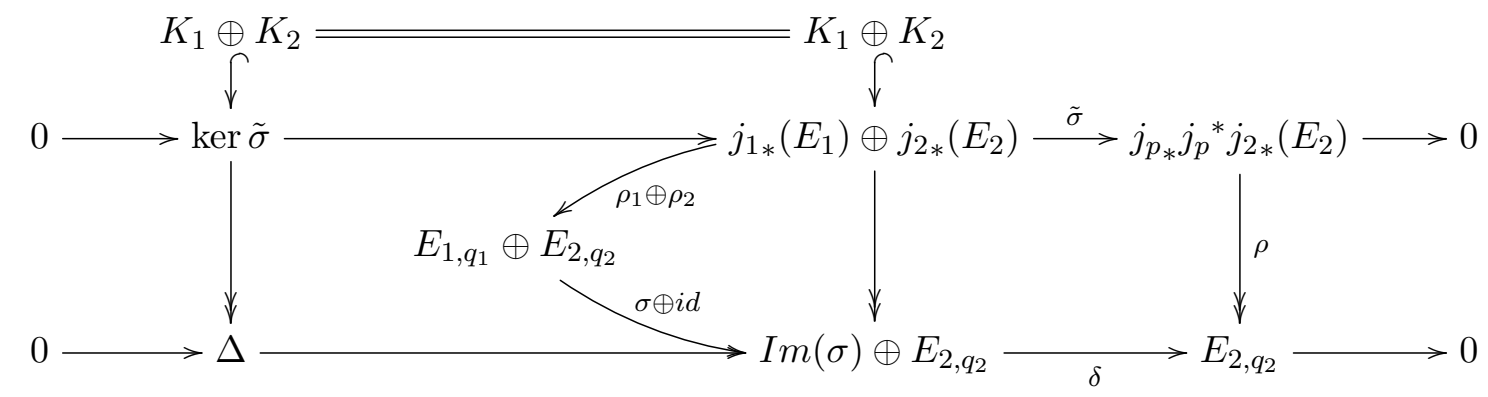

It follows immediately by construction that ker $\tilde{\sigma}$ is a sheaf of depth one on $C$, which coincides with $E_{i}$ on $C_{i} \backslash p$. One can easily see that the isomorphism class of ker $\tilde{\sigma}$ does not depend on the isomorphism classes of $E_{i}$. Moreover, the same happens if one uses $\sigma^{\prime}=\lambda \sigma$ with $\lambda \in \mathbb{C}^{*}$, instead of $\sigma$.

From now on, we will assume to be under the hypotesis of Lemma 3.1. Let $\mathbb{P}(\mathcal{F})$ be the projective bundle on $\mathcal{U}_{C_{1}}\left(r, d_{1}\right) \times \mathcal{U}_{C_{2}}\left(r, d_{2}\right)$. We can conclude that the construction of ker $\tilde{\sigma}$ depends on the data contained in $u=\left(\left(\left[E_{1}\right],\left[E_{2}\right]\right),[\sigma]\right) \in \mathbb{P}(\mathcal{F})$ and not on the particular choices of $E_{1}, E_{2}$ and $\sigma$.

Definition 3.1. We will denote by $E_{u}$ the kernel of $\tilde{\sigma}$ defined by $u \in \mathbb{P}(\mathcal{F})$.

The above construction gives the following:

Proposition 3.2. Let $E_{u}$ be the sheaf defined by $u=\left(\left(\left[E_{1}\right],\left[E_{2}\right]\right),[\sigma]\right) \in \mathbb{P}(\mathcal{F})$. Then $E_{u}$ is a depth one sheaf on $C$ with $\chi\left(E_{u}\right)=\chi\left(E_{1}\right)+\chi\left(E_{2}\right)-r$ and multirank $(r, r)$. It is a vector bundle if and only if $\sigma$ is an isomorphism. In this case, $E_{u \mid C_{i}}=E_{i}$.

Proof. Let $\operatorname{Rk}(\sigma)=k$. Since $E_{u}$ is a depth one sheaf, the stalk of $E_{u}$ at the node $p$ is isomorphic to

$$
\mathcal{O}_{p}^{a} \oplus \mathcal{O}_{q_{1}}^{b} \oplus \mathcal{O}_{q_{1}}^{c},
$$

where $a+b=\operatorname{Rk}\left(E_{u} \mid C_{1}\right)=r$ and $a+c=\operatorname{Rk}\left(E_{u} \mid C_{2}\right)=r$ (see Section 2). From the diagram 3.2 , it follows that the rank of the free part of the stalk of $E_{u}$ in $p$ is $k$, so $a=k$. Hence we have $\left.E_{u}\right|_{p} \simeq \mathcal{O}_{p}^{k} \oplus \mathcal{O}_{q_{1}}^{r-k} \oplus \mathcal{O}_{q_{2}}^{r-k}$. In particular, $E_{u}$ is a vector bundle if and only if $k=r$, i.e., exactly when $\sigma$ is an isomorphism.

In order to obtain a $w$-semistable sheaf, for some polarization $w$, we have the following necessary condition:

Lemma 3.3. Let $E=E_{u}$ be the sheaf defined by $u=\left(\left(\left[E_{1}\right],\left[E_{2}\right]\right),[\sigma]\right) \in \mathbb{P}(\mathcal{F})$ and let $k$ be the rank of $\sigma$. Then, if $E$ is $w$-semistable for some $w$, the following conditions are satisfied:

$$
\left\{\begin{array}{l}
\chi(E) w_{1} \leq \chi\left(E_{1}\right) \leq \chi(E) w_{1}+k \\
\chi(E) w_{2}+r-k \leq \chi\left(E_{2}\right) \chi(E) w_{2}+r
\end{array}\right.
$$

Proof. Assume that $E$ is $w$-semistable for a polarization $w$. Let $K_{1}$ be the kernel of the map

$$
\sigma \circ \rho_{1}: j_{1 *} E_{1} \rightarrow \operatorname{Im} \sigma
$$


and $K_{2}$ be the kernel of the map $\rho_{2}: j_{2 *} E_{2} \rightarrow E_{2, q_{2}}$, as in diagram 3.2. Since $K_{i}$ is a subsheaf of $E$, then by $w$-semistability of $E$ we must have $\mu_{w}\left(K_{i}\right) \leq \mu_{w}(E)$. We have: $\mu_{w}\left(K_{1}\right)=\frac{\chi\left(K_{1}\right)}{w_{1} r}=\frac{\chi\left(E_{1}\right)-k}{w_{1} r} \leq \frac{\chi(E)}{r}$, which implies

$$
\chi\left(E_{1}\right) \leq \chi(E) w_{1}+k .
$$

By replacing $\chi\left(E_{1}\right)=\chi(E)-\chi\left(E_{2}\right)+r$ in the above inequality, we obtain:

$$
\chi\left(E_{2}\right) \geq \chi(E) w_{2}+r-k .
$$

Finally, we have $\mu_{w}\left(K_{2}\right)=\frac{\chi\left(K_{2}\right)}{w_{2} r}=\frac{\chi\left(E_{2}\right)-r}{w_{2} r} \leq \frac{\chi(E)}{r}$, which implies

$$
\chi\left(E_{2}\right) \leq \chi(E) w_{2}+r .
$$

Again, by replacing $\chi\left(E_{2}\right)=\chi(E)-\chi\left(E_{1}\right)+r$ we obtain $\chi\left(E_{1}\right) \geq \chi(E) w_{1}$.

Given $u=\left(\left(\left[E_{1}\right],\left[E_{2}\right]\right),[\sigma]\right)$ and $E_{u}$ defined by $u$, we wonder if there exists a polarization $w$ such that the above conditions 3.3 hold. The answer depends only on numerical assumptions on $\left(\chi\left(E_{1}\right), \chi\left(E_{2}\right)\right)$ and $\operatorname{Rk} \sigma$ as it is shown in the following lemma.

Lemma 3.4. Let $r \geq 2$ and $1 \leq k \leq r$ be integers. There exists a non empty subset $\mathcal{W}_{r, k} \subset \mathbb{Z}^{2}$ such that for any pair $\left(\chi_{1}, \chi_{2}\right) \in \mathcal{W}_{r, k}$ we can find a polarization $w$ satisfying the following conditions:

$$
\left\{\begin{array}{l}
\chi w_{1} \leq \chi_{1} \leq \chi w_{1}+k \\
\chi w_{2}+r-k \leq \chi_{2} \leq \chi w_{2}+r
\end{array}\right.
$$

where $\chi=\chi_{1}+\chi_{2}-r$.

Proof. First of all note that if $\chi=0$, i.e. $\chi_{1}+\chi_{2}=r$ and we assume that $0 \leq \chi_{1} \leq r$, then any polarization $w$ satisfies conditions 3.4.

We distinguish two cases according to the sign of $\chi$. Assume that $\chi>0$. Then there exists a polarization $w$ satisfying conditions 3.4 , if and only if the following system has solutions:

$$
\left\{\begin{array}{l}
\frac{\chi_{1}-k}{\chi} \leq w_{1} \leq \frac{\chi_{1}}{\chi} \\
\frac{\chi_{2}-r}{\chi} \leq w_{2} \leq \frac{\chi_{2}+k-r}{\chi} \\
w_{1}+w_{2}=1 \\
0<w_{i}<1, w_{i} \in \mathbb{Q}
\end{array}\right.
$$

This occurs if and only if $\chi_{1}>0$ and $\chi_{2}>r-k$. Likewise, if $\chi<0$, then we have the following system:

$$
\left\{\begin{array}{l}
\frac{\chi_{1}}{\chi} \leq w_{1} \leq \frac{\chi_{1}-k}{\chi} \\
\frac{\chi_{2}-r+k}{\chi} \leq w_{2} \leq \frac{\chi_{2}-r}{\chi} \\
w_{1}+w_{2}=1 \\
0<w_{i}<1, w_{i} \in \mathbb{Q}
\end{array}\right.
$$

which has solutions if and only if $\chi_{1}<k$ and $\chi_{2}<r$. 


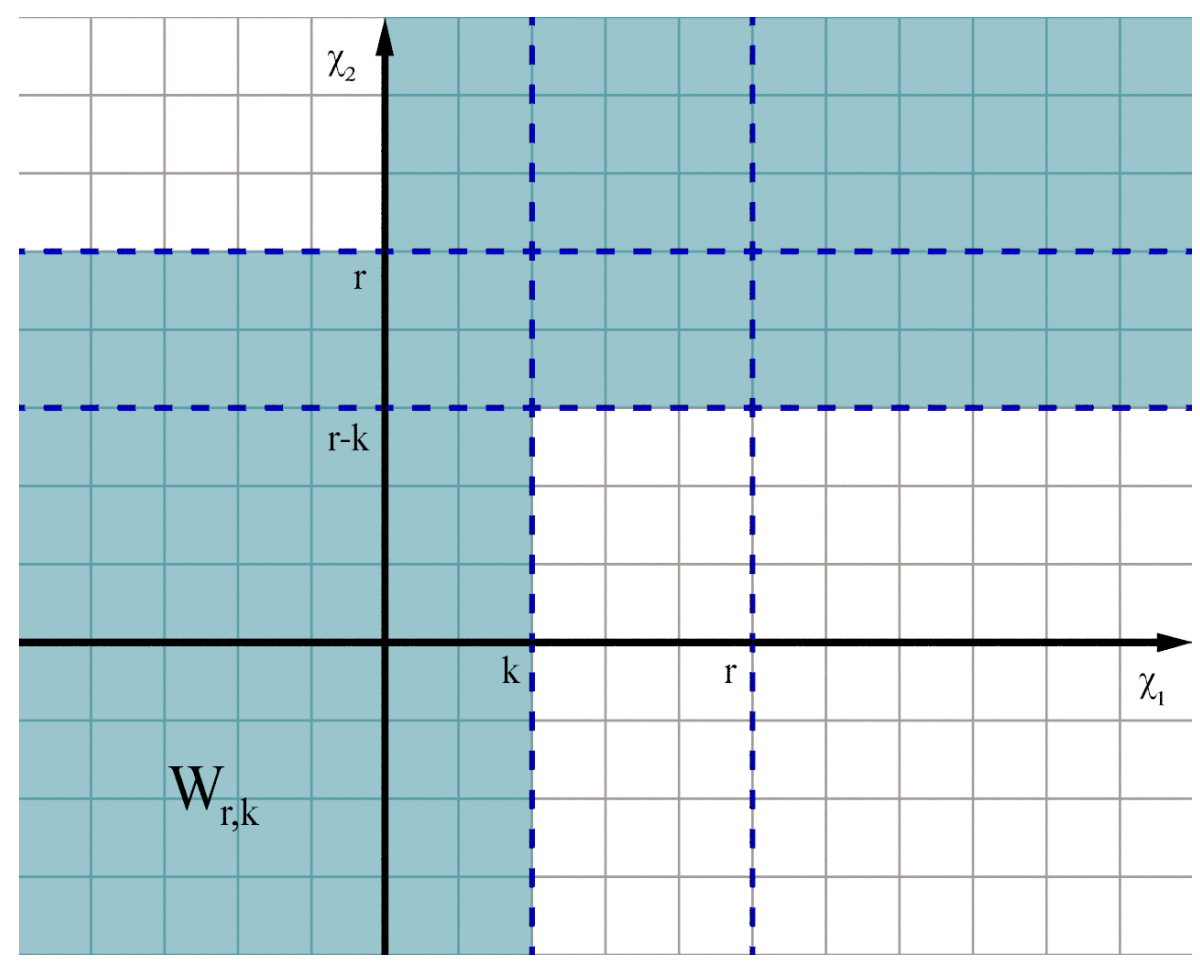

Remark 3.4.1. Let $\mathcal{W}_{r}=\bigcap_{k=1}^{r} \mathcal{W}_{r, k}$. Note that it is a non empty subset and it is actually $\mathcal{W}_{r, 1}$. Moreover, if $\left(\chi_{1}, \chi_{2}\right) \in \mathcal{W}_{r}$, then by the proof of lemma 3.4 it follows that we can find a polarization $w$ which satisfies the conditions 3.4 for all $k=1, \ldots, r$.

Assume that $\operatorname{Rk} \sigma=r$, i.e. $E$ is a vector bundle, then the necessary conditions of Lemma 3.3 are the same of Theorem 2.1. Hence, by the above Theorem, they are also sufficient to give $w$-semistability of $E$. So we obtain the following:

Corollary 3.5. Let $E=E_{u}$ be the sheaf defined by $u=\left(\left(\left[E_{1}\right],\left[E_{2}\right]\right),[\sigma]\right) \in \mathbb{P}(\mathcal{F})$. Assume that $\operatorname{Rk} \sigma=r$ and $\left(\chi\left(E_{1}\right), \chi\left(E_{2}\right)\right) \in \mathcal{W}_{r, r}$, then there exists a polarization $w$ such that $E$ is $w$-semistable. In particular, since $E_{i}$ are stable, then $E$ is w-stable too.

Unfortunately, when $E_{u}$ fails to be a vector bundle, the necessary conditions of Lemma 3.3 are not enough to ensure $w$-semistability, see [TiB11] for an example. Nevertheless, we are able to produce an open subset of $\mathcal{U}_{C_{1}}\left(r, d_{1}\right) \times \mathcal{U}_{C_{1}}\left(r, d_{1}\right)$ such that for every $u$ over this open, the sheaf $E_{u}$ is $w$-semistable.

We recall the following definition, see [NMRS78].

Definition 3.2. Let $G$ be a vector bundle on a smooth curve. For any integer $k$ we set:

$$
\mu_{k}(G)=\frac{\operatorname{deg}(G)+k}{r k(G)}
$$

A vector bundle $G$ is said $(m, k)$-semistable (resp. stable) if for any subsheaf $F$ we have:

$$
\mu_{m}(F) \leq \mu_{m-k}(G) \quad(\text { resp. }<) .
$$

Proposition 3.6. Let $E=E_{u}$ be the sheaf defined by $u=\left(\left(\left[E_{1}\right],\left[E_{2}\right]\right),[\sigma]\right) \in \mathbb{P}(\mathcal{F})$. Assume that $\operatorname{Rk} \sigma=k \leq r-1$. If $\left(\chi\left(E_{1}\right), \chi\left(E_{2}\right)\right) \in \mathcal{W}_{r, k}, E_{1}$ is $(0, k)$-semistable and $E_{2}$ is $(0, r)$-semistable, then 
there exists a polarization $w$ such that $E$ is w-semistable. Moreover, if $E_{1}$ is $(0, k)$-stable or $E_{2}$ is $(0, r)$ - stable then $E$ is w-stable too.

Proof. Since $\left(\chi\left(E_{1}\right), \chi\left(E_{2}\right)\right) \in \mathcal{W}_{r, k}$, by Lemma 3.4 there exists a polarization $w$ such that the necessary conditions 3.3 hold. We claim that if $E_{1}$ is $(0, k)$-semistable and $E_{2}$ is $(0, r)$-semistable, then $E$ is $w$ semistable.

Let $F \subset E$ be a subsheaf, it is a sheaf of depth one too. Assume that $F$ has multirank $\left(s_{1}, s_{2}\right)$ and that at the node $p$ the stalk of $F$ is $\mathcal{O}_{p}^{s} \oplus \mathcal{O}_{q_{1}}^{a} \oplus \mathcal{O}_{q_{2}}^{b}$, with $s \geq 0, s_{1}=s+a \leq r$ and $s_{2}=s+b \leq r$. Since $\operatorname{Rk} \sigma=k$, by construction the free part of the stalk of $E$ at $p$ is $\mathcal{O}_{p}^{k}$. This implies that $0 \leq s \leq k$.

By construction, there exists two vector bundles $F_{1} \subseteq E_{1}$ and $F_{2} \subseteq E_{2}$ such that $F$ is the kernel of the restriction of $\tilde{\sigma}$ to the subsheaf $j_{1 *}\left(F_{1}\right) \oplus j_{2 *}\left(F_{2}\right)$ :

$$
\tilde{\sigma}_{\mid j_{1 *}\left(F_{1}\right) \oplus j_{2 *}\left(F_{2}\right)}: j_{1_{*}}\left(F_{1}\right) \oplus j_{2 *}\left(F_{2}\right) \rightarrow j_{p_{*}} j_{p}{ }^{*} j_{2 *}\left(E_{2}\right) .
$$

By proceding as in the diagram 3.2, we deduce that $F$ fits into an exact sequence as follows:

$$
0 \rightarrow G_{1} \oplus G_{2} \rightarrow F \rightarrow \mathbb{C}_{p}^{s} \rightarrow 0,
$$

where $G_{1}$ is the kernel of $\left.\left(\sigma \circ \rho_{1}\right)\right|_{F_{1}}$ and $G_{2}$ is the kernel of $\left.\rho_{2}\right|_{F_{2}}$. Hence $G_{i} \subseteq K_{i}$. Note that if $s=0$, then actually $F \simeq G_{1} \oplus G_{2}$.

For any $s$, we compute the $w$-slope of $F$ :

$$
\begin{aligned}
& \mu_{w}(F)=\frac{\chi(F)}{w_{1} s_{1}+w_{2} s_{2}}=\frac{\chi\left(G_{1}\right)+\chi\left(G_{2}\right)+s}{w_{1} s_{1}+w_{2} s_{2}}= \\
= & \frac{\operatorname{deg}\left(G_{1}\right)+s_{1}\left(1-g_{1}\right)+\operatorname{deg}\left(G_{2}\right)+s_{2}\left(1-g_{2}\right)+s}{w_{1} s_{1}+w_{2} s_{2}} .
\end{aligned}
$$

Since $E_{1}$ is $(0, k)$-semistable, then we have:

$$
\frac{\operatorname{deg}\left(G_{1}\right)}{s_{1}} \leq \frac{d_{1}-k}{r}
$$

since $E_{2}$ is $(0, r)$-semistable, then $E_{2}\left(-q_{2}\right)$ is $(0, r)$-semistable too, so we have:

$$
\frac{\operatorname{deg}\left(G_{2}\right)}{s_{2}} \leq \frac{d_{2}-2 r}{r}
$$

By replacing we obtain:

$$
\begin{array}{r}
\mu_{w}(F) \leq \frac{1}{w_{1} s_{1}+w_{2} s_{2}}\left[s_{1} w_{1}\left(\frac{\left(d_{1}-k\right)+r\left(1-g_{1}\right)}{w_{1} r}\right)+s_{2} w_{2}\left(\frac{\left(d_{2}-r\right)+r\left(1-g_{2}\right)}{w_{2} r}\right)+s-s_{2}\right]= \\
=\frac{s_{1} w_{1}}{w_{1} s_{1}+w_{2} s_{2}} \mu_{w}\left(K_{1}\right)+\frac{s_{2} w_{2}}{w_{1} s_{1}+w_{2} s_{2}} \mu_{w}\left(K_{2}\right)+\frac{s-s_{2}}{w_{1} s_{1}+w_{2} s_{2}} .
\end{array}
$$

By Lemma 3.3, we have that $\mu_{w}\left(K_{i}\right) \leq \mu_{w}(E)$, so we obtain:

$$
\mu_{w}(F) \leq \mu_{w}(E)+\frac{s-s_{2}}{w_{1} s_{1}+w_{2} s_{2}} .
$$

Since $s-s_{2} \leq 0$, we have that $\mu_{w}(F) \leq \mu_{w}(E)$.

Finally, if $E_{1}$ is $(0, k)$-stable or $E_{2}$ is $(0, r)$-stable, then the above inequality is strict. This concludes the proof.

Note that, by definition, if $E_{i}$ is $(0, r)$-stable, then it is also $(0, k)$-stable for all $k \leq r$. 
Lemma 3.7. Let $\mathcal{U}_{C_{i}}\left(r, d_{i}\right)$ be the moduli space of semistable vector bundles of rank $r$ and degree $d_{i}$ on a smooth curve $C_{i}$ of genus $g_{i}$. If $d_{i}$ and $r$ are coprime and $g_{i}>r+1$, then the locus of vector bundles of $\mathcal{U}_{C_{i}}\left(r, d_{i}\right)$ which are $(0, r)$-stable is a non empty open subset of $\mathcal{U}_{C_{i}}\left(r, d_{i}\right)$.

Proof. Let us consider the locus

$$
Y=\left\{[E] \in \mathcal{U}_{C_{i}}\left(r, d_{i}\right) \mid E \text { is not }(0, r)-\text { stable }\right\} .
$$

We can consider the subset $Y_{a, s}$ of $Y$ given by all stable vector bundles $E$ which can be written as

$$
0 \rightarrow F \rightarrow E \rightarrow Q \rightarrow 0
$$

where $F$ is a subbundle of $E$ with $\operatorname{deg}(F)=a$ and $\operatorname{Rk}(F)=s \leq r-1$ and

$$
\mu(E)-1=\mu_{-r}(E) \leq \mu(F) \leq \mu_{0}(E)=\mu(E) .
$$

By a deformation argument (see the proof of Proposition 1.4 of [RTiB99]), one can prove that if $Y_{a, s} \neq \emptyset$ then for a general $E$ in $Y_{a, s}$ both $F$ and $Q$ are stable. Moreover, since $E$ is stable, we have $\operatorname{Hom}(Q, F)=0$. Hence we can write

$$
\begin{gathered}
\operatorname{dim} Y_{a, s} \leq \operatorname{dim} \mathcal{U}_{C_{i}}(s, a)+\operatorname{dim} \mathcal{U}_{C_{i}}\left(r-s, d_{i}-a\right)+\operatorname{dim} H^{1}\left(C_{i}, \mathcal{H o m}(Q, F)\right)-1= \\
=\left(g_{i}-1\right)\left(r^{2}-r s+s^{2}\right)+1+\left(d_{i} s-a r\right) .
\end{gathered}
$$

Hence:

$$
\operatorname{dim} \mathcal{U}_{C_{i}}\left(r, d_{i}\right)-\operatorname{dim} Y_{a, s} \geq\left(g_{i}-1\right)\left(r s-s^{2}\right)-\left(d_{i} s-a r\right) .
$$

Since $E \in Y, \mu_{0}(F) \geq \mu_{-r}(E)$, i.e.

$$
\frac{a}{s} \geq \frac{d_{i}-r}{r}
$$

which implies

$$
d_{i} s-a r \leq r s .
$$

Finally, if $g_{i}>1+r$, for all $s \leq r-1$, we have:

$$
\operatorname{dim} \mathcal{U}_{C_{i}}\left(r, d_{i}\right)-\operatorname{dim} Y_{a, s} \geq s\left[\left(g_{i}-1\right)(r-s)-r\right]>0
$$

which concludes the proof.

\section{MAin RESUlts}

In this section we prove our main results. We assume that the hypothesis of Lemma 3.1 are satisfied.

Let $\mathbb{P}(\mathcal{F})$ be the projective bundle on $\mathcal{U}_{C_{1}}\left(r, d_{1}\right) \times \mathcal{U}_{C_{2}}\left(r, d_{2}\right)$. For each $1 \leq k \leq r-1$, let $\mathcal{B}_{k}$ be the subset of $\mathbb{P}(\mathcal{F})$ such that

$$
\mathcal{B}_{k} \cap \pi^{-1}\left(\left[E_{1}\right],\left[E_{2}\right]\right)=\left\{[\sigma] \in \mathbb{P}\left(\operatorname{Hom}\left(E_{1, q_{1}}, E_{2, q_{2}}\right)\right) \mid \operatorname{Rk}(\sigma) \leq k\right\} .
$$

It is a proper closed subvariety of $\mathbb{P}(\mathcal{F})$.

Definition 4.1. We will denote by $\mathscr{U}$ the open subset given by the complement of $\mathcal{B}_{r-1}$ in $\mathbb{P}(\mathcal{F})$. 
Remark 4.0.1. Note that $\operatorname{dim} \mathscr{U}=\operatorname{dim} \mathbb{P}(\mathcal{F})=r^{2}\left(g_{1}+g_{2}-1\right)+1$. If we denote by $\pi_{\mathscr{U}}$ the restriction of $\pi$ to $\mathscr{U}$ we have that, by construction,

$$
\pi_{\mathscr{U}}: \mathscr{U} \rightarrow \mathcal{U}_{C_{1}}\left(r, d_{1}\right) \times \mathcal{U}_{C_{2}}\left(r, d_{2}\right)
$$

is a fiber bundle whose fibers are isomorphic to $\mathrm{PGL}(r)$. More precisely

$$
\pi_{\mathscr{U}}^{-1}\left(\left[E_{1}\right],\left[E_{2}\right]\right)=\mathbb{P}\left(\operatorname{GL}\left(E_{1, q_{1}}, E_{2, q_{2}}\right)\right) .
$$

For $\chi=d_{1}+d_{2}+r\left(1-g_{1}-g_{2}\right)$, let $\mathcal{U}_{C}(w, r, \chi)_{d_{1}, d_{2}}$ be the irreducible component of the moduli space of depth one sheaves on $C$ of rank $r$ and characteristic $\chi$ corresponding to the multidegree $\left(d_{1}, d_{2}\right)$, see Section 2. Let $\mathcal{V}_{C}(w, r, \chi)_{d_{1}, d_{2}} \subset \mathcal{U}_{C}(w, r, \chi)_{d_{1}, d_{2}}$ be the subset parametrizing classes of vector bundles.

Theorem 4.1. Let $C$ be a nodal curve with a single node $p$ and two smooth irreducible components $C_{i}$ of genus $g_{i} \geq 1$. Fix $r \geq 2$, for any $d_{i} \in \mathbb{Z}$ we set $\chi_{i}=d_{i}+r\left(1-g_{i}\right)$ and $\chi=d_{1}+d_{2}+r\left(1-g_{1}-g_{2}\right)$. Assume that $r$ is coprime with both $d_{1}$ and $d_{2}$ and $\left(\chi_{1}, \chi_{2}\right) \in \mathcal{W}_{r, r}$. Then there exists a polarization $w$ such that the map

$$
\phi: \mathbb{P}(\mathcal{F})-->\mathcal{U}_{C}(w, r, \chi)_{d_{1}, d_{2}}
$$

sending $u \rightarrow\left[E_{u}\right]$ is birational. In particular, the restriction $\left.\phi\right|_{\mathscr{U}}$ is a an injective morphism and the image $\Phi(\mathscr{U})$ is contained in $\mathcal{V}_{C}(w, r, \chi)_{d_{1}, d_{2}}$.

Proof. Let $u=\left(\left(\left[E_{1}\right],\left[E_{2}\right]\right),[\sigma]\right) \in \mathbb{P}(\mathcal{F})$ and consider the sheaf $E=E_{u}$ defined by $u$, as in Section 3. Since $\left(\chi_{1}, \chi_{2}\right) \in \mathcal{W}_{r, r}$, then, as a consequence of Lemma 3.4 and Corollary 3.5 there exists a polarization $w$ such that $E_{u}$ is $w$-semistable for every $u \in \mathscr{U}$. This gives a point in the moduli space $\mathcal{U}_{C}(w, r, \chi)_{d_{1}, d_{2}}$ and it shows that $\phi$ is well defined at least on $\mathscr{U}$.

Now we will prove that $\phi_{\mid \mathscr{U}}$ is injective. Let $u=\left(\left(\left[E_{1}\right],\left[E_{2}\right]\right),[\sigma]\right)$ and $u^{\prime}=\left(\left(\left[E_{1}^{\prime}\right],\left[E_{2}^{\prime}\right]\right),\left[\sigma^{\prime}\right]\right)$ in $\mathscr{U}$ with $\phi(u)=[E]$ and $\phi\left(u^{\prime}\right)=\left[E^{\prime}\right]$. Assume that $\phi(u)=\phi\left(u^{\prime}\right)$. Since $E$ and $E^{\prime}$ are both $w$-stable and are in the the same $S_{w}$-equivalence class, then they have to be isomorphic (see Section 2). Let $\tau: E \rightarrow E^{\prime}$ be an isomorphism. This induces an isomorphism $\tau_{i}: E_{i} \rightarrow E_{i}^{\prime}$. So we can assume, that $E_{i}^{\prime}=E_{i}$ and, thus $\sigma, \sigma^{\prime}: E_{1, q_{1}} \rightarrow E_{2, q_{2}}$ and $\tau_{i}: E_{i} \rightarrow E_{i}$ are isomorphism. As $E_{p}$ (respectively $E_{p}^{\prime}$ ) is obtained by glueing $E_{1, q_{1}}$ with $E_{2, q_{2}}$ along the isomorphism $\sigma$ (respectively along $\sigma^{\prime}$ ), $\tau_{i}$ have to satisfy a compatibility condition, summarized in the following commutative diagram:

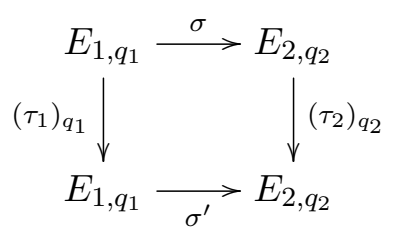

Since $E_{i}$ is stable we have $\operatorname{Hom}\left(E_{i}, E_{i}\right) \simeq \mathbb{C} \cdot i d_{E_{i}}$. Hence $\left(\tau_{i}\right)_{q_{i}}$ is the multiplication by some $\lambda_{i} \in \mathbb{C}^{*}$. In particular, $\sigma^{\prime}$ is a non zero multiple of $\sigma$ and thus $[\sigma]=\left[\sigma^{\prime}\right]$.

Now we prove that $\phi_{\mid \mathscr{U}}$ is a morphism. It is enough to prove that $\phi$ is regular at $u_{0}$, for any $u_{0} \in \mathscr{U}$. At this hand, we claim that there exists a non empty open subset $W \subseteq \mathscr{U}$ with $u_{0} \in W$ and a vector bundle $\mathcal{E}$ on $W \times C$ such that

$$
\left[\left.\mathcal{E}\right|_{u \times C}\right]=\phi(u), \quad \forall u \in W .
$$

Step 1: There exist two sheaves $\mathcal{Q}$ and $\mathcal{R}$ on $\mathscr{U} \times C$ such that, for each $u=\left(\left(\left[E_{1}\right],\left[E_{2}\right],[\sigma]\right)\right.$, with $u \in \mathscr{U}$, we have

$$
\left.\mathcal{Q}\right|_{u \times C} \simeq j_{1 *}\left(E_{1}\right) \oplus j_{2 *}\left(E_{2}\right), \quad \mathcal{R}_{\mid u \times C} \simeq j_{p_{*}}\left(j_{p}{ }^{*}\left(j_{2 *}\left(E_{2}\right)\right)\right),
$$


where $j_{p}: p \hookrightarrow C$ and $j_{i}: C_{i} \hookrightarrow C$ are the natural inclusions.

Consider the diagram

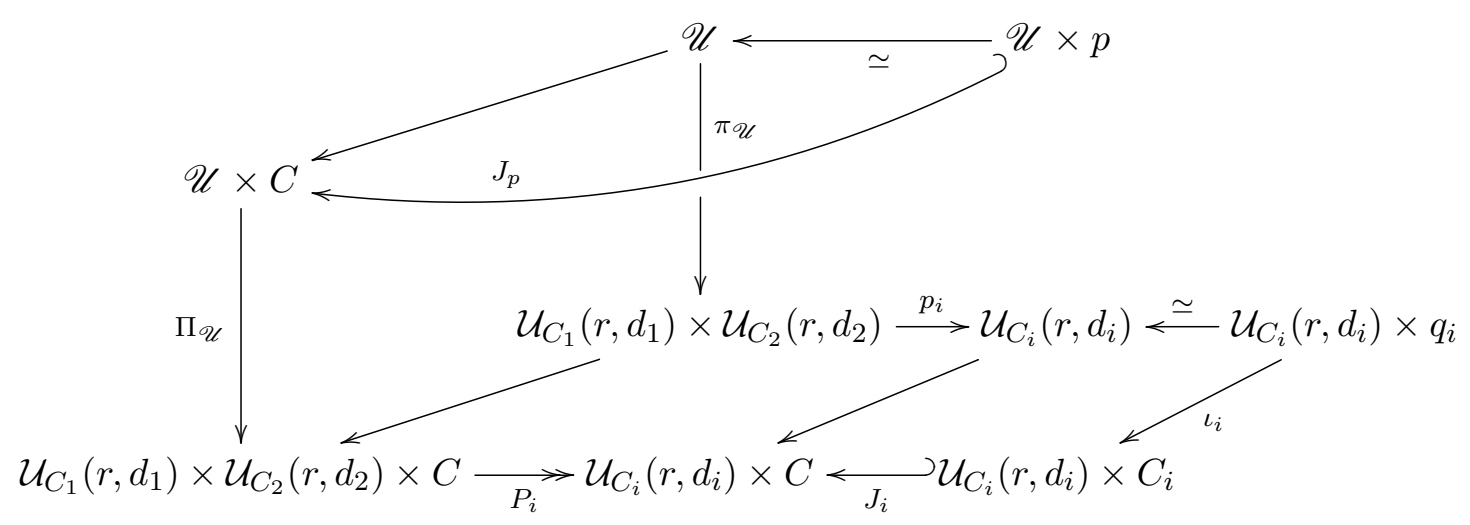

where the morphisms which appear have been defined as

$$
J_{i}=i d_{\mathcal{U}_{C_{i}}\left(r, d_{i}\right)} \times j_{i}, \quad P_{i}=p_{i} \times i d_{C}, \quad \Pi_{\mathscr{U}}=\pi_{\mathscr{U}} \times i d_{C}, \quad J_{p}=i d_{\mathscr{U}} \times j_{p} .
$$

If, as before, we denote with $\mathcal{P}_{i}$ the Poincaré bundle on $\mathcal{U}_{C_{i}}\left(r, d_{i}\right) \times C_{i}$ we can set

$$
\mathcal{Q}_{i}=\Pi_{\mathscr{U}}^{*}\left(P_{i}^{*}\left(J_{i *}\left(\mathcal{P}_{i}\right)\right)\right) \quad \mathcal{Q}=\mathcal{Q}_{1} \oplus \mathcal{Q}_{2},
$$

and

$$
\mathcal{R}=J_{p_{*}}\left(J_{p}{ }^{*}\left(Q_{2}\right)\right) .
$$

Note that $\operatorname{Supp}(\mathcal{R})=\mathscr{U} \times p$. Moreover, one can verify that if we identify $\mathscr{U} \times p$ with $\mathscr{U}$ we have:

$$
J_{p}^{*}\left(\mathcal{Q}_{i}\right) \simeq \pi_{\mathscr{U}}^{*}\left(p_{i}^{*}\left(\iota_{i}^{*} \mathcal{P}_{i}\right)\right),
$$

where $\iota_{i}: \mathcal{U}_{C_{i}}\left(r, d_{i}\right) \times q_{i} \hookrightarrow \mathcal{U}_{C_{i}}\left(r, d_{i}\right) \times C_{i}$.

Step 2: There is an open subset $W \subset \mathscr{U}$ containing $u_{0}$ and a surjective map of sheaves

$$
\left.\left.\mathcal{Q}_{1} \oplus \mathcal{Q}_{2}\right|_{W \times C} \stackrel{\Sigma_{W}}{\longrightarrow} \mathcal{R}\right|_{W \times C}
$$

whose kernel is the desired vector bundle $\mathcal{E}$ on $W \times C$.

Let $\pi: \mathbb{P}(\mathcal{F}) \rightarrow \mathcal{U}_{C_{1}}\left(r, d_{1}\right) \times \mathcal{U}_{C_{2}}\left(r, d_{2}\right)$ be the projective bundle defined in Lemma 3.1. First of all consider on $\mathbb{P}(\mathcal{F})$ the tautological line bundle $\mathcal{O}_{\mathbb{P}(\mathcal{F})}(-1)$ which is, by definition, the subsheaf of $\pi^{*}(\mathcal{F})$ whose fiber at $u \in \mathbb{P}(\mathcal{F})$ is

$$
\operatorname{Span}(\sigma) \subset \operatorname{Hom}\left(E_{1, q_{1}}, E_{2, q_{2}}\right),
$$

where $u=\left(\left(\left[E_{1}\right],\left[E_{2}\right]\right),[\sigma]\right)$. We can choose $W$ to be an open subset of $\mathscr{U}$ containing the point $u_{0}$ and admitting a section $s \in \mathcal{O}_{\mathbb{P}(\mathcal{F})}(-1)(W)$ with $s(u) \neq 0$, for any $u \in W$.

In particular $s$ induces a map of sheaves

$$
\left.\left.s: \pi_{\mathscr{U}}^{*} p_{1}^{*}\left(\iota_{1}^{*}\left(\mathcal{P}_{1}\right)\right)\right)\left.\right|_{W} \rightarrow \pi_{\mathscr{U}}^{*} p_{2}^{*}\left(\iota_{2}^{*}\left(\mathcal{P}_{2}\right)\right)\right)\left.\right|_{W} .
$$

such that $s_{u}: E_{1, q_{1}} \rightarrow E_{2, q_{2}}$ is an isomorphism and $\left[s_{u}\right]=[\sigma]$ in $\mathbb{P}\left(\operatorname{Hom}\left(E_{1, q_{1}}, E_{2, q_{2}}\right)\right)$.

We can also define a morphism of sheaves

$$
\left.\left.\left.s-i d_{2}: \pi_{\mathscr{U}}^{*} p_{1}^{*}\left(\iota_{1}^{*}\left(\mathcal{P}_{1}\right)\right)\right)\left.\right|_{W} \oplus \pi_{\mathscr{U}}^{*} p_{2}^{*}\left(\iota_{2}^{*}\left(\mathcal{P}_{2}\right)\right)\right)\left.\right|_{W} \rightarrow \pi_{\mathscr{U}}^{*} p_{2}^{*}\left(\iota_{2}^{*}\left(\mathcal{P}_{2}\right)\right)\right)\left.\right|_{W}
$$

where $i d_{2}$ is the identity of $\left.\pi_{\mathscr{U}}^{*} p_{2}^{*}\left(\iota_{2}^{*}\left(\mathcal{P}_{2}\right)\right)\right)\left.\right|_{W}$. 
This allows us to define the map $\Sigma_{W}$ we are looking for. Indeed, since $\operatorname{Supp}\left(\left.\mathcal{R}\right|_{W \times C}\right)=W \times p$, it is enough to give the map on $W \times p$, which can be identified with $W$. Using the isomorphism 4.3, we have a diagram which defines $\Sigma_{W}$ :

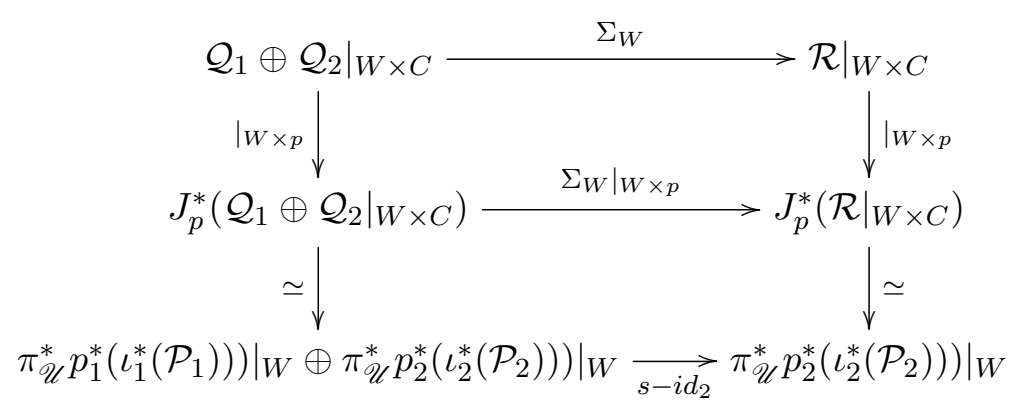

By taking the kernel $\mathcal{E}$ of this map we concludes the second step of the proof of the claim. In particular, $\phi_{\mid \mathscr{U}}$ is a morphism.

By construction, $\phi(\mathscr{U})$ is contained in $\mathcal{V}_{C}(w, r, \chi)_{d_{1}, d_{2}}$ and it coincide with the open subset of $w$ semistable vector bundles whose restrictions are semistable. Moreover, $\mathcal{V}_{C}(w, r, \chi)_{d_{1}, d_{2}}$ is a dense open subset of $\mathcal{U}_{C}(w, r, \chi)_{d_{1}, d_{2}}$ (see [Ses82]). Notice that, by 4.0.1, we have:

$$
\operatorname{dim}(\phi(\mathscr{U}))=\operatorname{dim}(\mathscr{U})=r^{2}\left(g_{1}+g_{2}-1\right)+1
$$

which is the dimension of $\mathcal{U}_{C}(w, r, \chi)_{d_{1}, d_{2}}$, see Theorem 2.1. This implies that $\phi$ is a dominant map. Hence, by a generic smoothness argument, we can conclude that $\phi_{\mid \mathscr{U}}$ is a birational morphism.

Corollary 4.2. Let $C$ be a nodal curve with a single node $p$ and two smooth irreducible components $C_{i}$ of genus $g_{i} \geq 1$. Assume that the moduli space $\mathcal{U}_{C}(w, r, \chi)$ has an irreducible component corresponding to bidegree $\left(d_{1}, d_{2}\right)$ with $d_{1}$ and $d_{2}$ coprime with $r$. Then this component is birational to a projective bundle over the smooth variety $\mathcal{U}_{C_{1}}\left(r, d_{1}\right) \times \mathcal{U}_{C_{2}}\left(r, d_{2}\right)$.

Note that $\phi$ provides a desingularization of the component $\mathcal{U}_{C}(w, r, \chi)_{d_{1}, d_{2}}$.

If the genus of the curve $C_{i}$ is big enough, we can be more precise about the domain of the rational map $\phi$.

Assume that $g_{i}>r+1$, then by Lemma 3.7 the locus of vector bundles of $\mathcal{U}_{C_{i}}\left(r, d_{i}\right)$ which are $(0, r)$-stable is a non empty open subset of $\mathcal{U}_{C_{i}}\left(r, d_{i}\right)$, let us denote it by $V_{i}$.

Definition 4.2. We will denote by $\mathscr{V}$ the open subset $\pi^{-1}\left(V_{1} \times V_{2}\right)$ in $\mathbb{P}(\mathcal{F})$.

By construction, $\mathscr{V}$ is a projective bundle over $V_{1} \times V_{2}$.

Theorem 4.3. Assume that the hypothesis of Theorem 4.1 hold. Moreover, assume that $g_{i}>r+1$ and $\left(\chi_{1}, \chi_{2}\right) \in \mathcal{W}_{r}$. Then there exists a polarization $w$ such that the map $\phi$ sending $u$ to $\left[E_{u}\right]$ is a birational map such that $\left.\phi\right|_{\mathscr{U} \cup \mathscr{V}}$ is a morphism.

Proof. Since $\left(\chi_{1}, \chi_{2}\right) \in \mathcal{W}_{r}$ then, by Remark 3.4.1, there exists a polarization $w$ such that conditions 3.4 hold for any $k=1, \cdots, r$. In particular, as $\mathcal{W}_{r} \subset \mathcal{W}_{r, r}$, Theorem 4.1 holds: $\phi$ is a birational map which is defined on the open subset $\mathscr{U}$.

Assume that $u \in \mathscr{V}$ and $u \notin \mathcal{U}$. Then $u=\left(\left(\left[E_{1}\right],\left[E_{2}\right]\right),[\sigma]\right)$, with $\left(\left[E_{1}\right],\left[E_{2}\right]\right) \in V_{1} \times V_{2}$ and $\operatorname{Rk} \sigma \leq r-1$. Since $\left[E_{i}\right] \in V_{i}$, then by lemma 3.6, $E_{u}$ is $w$-semistable, hence $\phi$ is defined all over the open subset $\mathscr{V}$ too. 
To prove that $\left.\phi\right|_{\mathscr{V}}$ is a morphism, we can proceed as in the proof of Theorem 4.1, just by replacing $\mathscr{U}$ with $\mathscr{V}$ and $\mathcal{U}_{C_{i}}\left(r, d_{i}\right)$ with $V_{i}$.

\section{Fixed-Determinant moduli SPACE}

Let $C$ be a smooth curve of genus $g \geq 1$ and $L \in \operatorname{Pic}^{d}(C)$. We recall that the moduli space of semistable vector bundles of rank $r$ and determinant $L$ on $C$ is denoted by $\mathcal{S} U_{C}(r, L)$ and it is an irreducible and projective variety. It is the fiber of the determinant map:

$$
\operatorname{det}: \mathcal{U}_{C}(r, d) \rightarrow \operatorname{Pic}^{d}(C) \text {. }
$$

In this section we will investigate a similar subvariety of the moduli space $\mathcal{U}_{C}(w, r, \chi)_{d_{1}, d_{2}}$ for a nodal reducible curve with two irreducible component $C_{i}$. Fix a pair $\left(L_{1}, L_{2}\right)$ with $L_{i} \in \operatorname{Pic}^{d_{i}}\left(C_{i}\right)$. Note that there exists a unique line bundle $L$ on the nodal curve $C$ whose restriction to the component $C_{i}$ is $L_{i}$. Recall that $\mathcal{V}_{C}(w, r, \chi)_{d_{1}, d_{2}} \subset \mathcal{U}_{C}(w, r, \chi)_{d_{1}, d_{2}}$ is the open subset parametrizing $w$-semistable classes which are represented by vector bundles.

Definition 5.1. Let $L$ be the line bundle on $C$ induced by the pair $\left(L_{1}, L_{2}\right)$. We define $\mathcal{S} U_{C}(w, r, L)$ as the closure of

$$
\left\{[E] \in \mathcal{V}_{C}(w, r, \chi)_{d_{1}, d_{2}} \mid \operatorname{det} E=L\right\}
$$

in $\mathcal{U}_{C}(w, r, \chi)_{d_{1}, d_{2}}$.

If we assume that $r$ and $d_{i}$ are coprime, then $\mathcal{S} U_{C_{i}}\left(r, L_{i}\right)$ is a smooth irreducible projective variety of dimension $\left(r^{2}-1\right)\left(g_{i}-1\right)$. As in Lemma 3.1, we can define a vector bundle $\mathcal{F}_{L}$ on $\mathcal{S U}_{C_{1}}\left(r, L_{1}\right) \times$ $\mathcal{S} U_{C_{2}}\left(r, L_{2}\right)$ just by restricting $\mathcal{F}$. Then we can consider the associated projective bundle $\mathbb{P}\left(\mathcal{F}_{L}\right)$ and

$$
\mathscr{U}_{L}=\mathscr{U} \cap \mathbb{P}\left(\mathcal{F}_{L}\right),
$$

a PGL $(r)$-bundle on $\mathcal{S} U_{C_{1}}\left(r, L_{1}\right) \times \mathcal{S} U_{C_{2}}\left(r, L_{2}\right)$. We denote by $\phi_{L}$ the restriction of the morphism $\phi$ defined in Theorem 4.1 to $\mathscr{U}_{L}$. As a consequences of Theorem 4.1, we have the following:

Corollary 5.1. In the hypothesis of Theorem 4.1, the map

$$
\phi_{L}: \mathbb{P}\left(\mathcal{F}_{L}\right)-->\mathcal{S} U_{C}(w, r, L)
$$

is a birational map, whose restriction $\left.\phi_{L}\right|_{\mathscr{U}_{L}}$ is an injective morphism.

Proof. Note that $\left.\phi_{L}\right|_{\mathscr{U}_{L}}$ is a morphism and its image is the following subset:

$$
\operatorname{Im} \phi_{L}=\left\{E \in \mathcal{V}_{C}(w, r, \chi)_{d_{1}, d_{2}} \mid\left[E_{\mid C_{i}}\right] \in \mathcal{S} U_{C_{i}}\left(r, L_{i}\right)\right\} .
$$

In particular, $\operatorname{Im} \phi_{L} \subseteq \mathcal{S} U_{C}(w, r, L)$.

Consider the following map:

$$
\psi: \mathcal{V}_{C}(w, r, \chi)_{d_{1}, d_{2}} \rightarrow \operatorname{Pic}^{d_{1}}\left(C_{1}\right) \times \operatorname{Pic}^{d_{2}}\left(C_{2}\right),
$$

sending $E \rightarrow\left(\operatorname{det}\left(\left.E\right|_{C_{1}}\right), \operatorname{det}\left(\left.E\right|_{C_{2}}\right)\right)$, which fit into the following commutative diagramm:

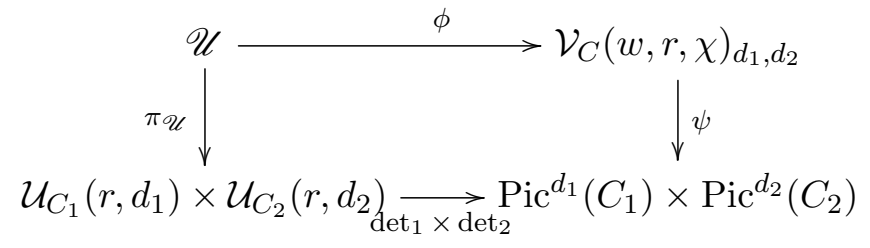


It follows immediately that $\psi$ is a surjective morphism and $\operatorname{Im} \phi_{L} \subset \psi^{-1}\left(L_{1}, L_{2}\right)$.

We claim that $\psi$ has irreducible fibers of dimension $\left(r^{2}-1\right)\left(g_{1}+g_{2}-1\right)$.

First of all we prove that any two fibers of $\psi$ are isomorphic. Let $\left(L_{1}, L_{2}\right)$ and $\left(L_{1}^{\prime}, L_{2}^{\prime}\right)$ in $\operatorname{Pic}^{d_{1}}\left(C_{1}\right) \times$ $\operatorname{Pic}^{d_{2}}\left(C_{2}\right)$, then there exists $\xi_{i} \in \operatorname{Pic}^{0}\left(C_{i}\right)$ such that $L_{i} \otimes \xi_{i}^{r} \simeq L_{i}^{\prime}$. Let $\xi$ be the unique line bundle on $C$ such that $\xi_{\mid C_{i}} \simeq \xi_{i}$. The natural map

$$
\psi^{-1}\left(L_{1}, L_{2}\right) \rightarrow \psi^{-1}\left(L_{1}^{\prime}, L_{2}^{\prime}\right)
$$

sending $E$ to $E \otimes \xi$ preserves $w$-semistability and it gives an isomorphism of the fibers. In particular, from fiber dimension Theorem (see [Har77], p.95), this implies that any fiber has pure dimension $\left(r^{2}-1\right)\left(g_{1}+g_{2}-1\right)$.

Finally we prove that any fiber is irreducible. Let $Y=\mathcal{V}_{C}(w, r, \chi)_{d_{1}, d_{2}} \backslash \phi(\mathscr{U})$, it is a proper subvariety of $\mathcal{V}_{C}(w, r, \chi)_{d_{1}, d_{2}}$. Assume that the fiber of $\psi$ over $\left(L_{1}, L_{2}\right)$ is reducible. Let $F_{1}$ be the irreducible component containing $\phi\left(\mathscr{U}_{L}\right)$, then there exists an irreducible component $F_{2} \subset Y$. So the restriction of $\psi$ to $Y$ is a surjective morphism whose fibers have dimension $\left(r^{2}-1\right)\left(g_{1}+g_{2}-1\right)$. This implies that $\operatorname{dim} Y=\operatorname{dim} \mathcal{V}_{C}(w, r, \chi)_{d_{1}, d_{2}}$, which is impossible.

This allows us to conclude that $\mathcal{S} U_{C}(w, r, L)$ is irreducible too and $\phi_{L}$ is a birational morphism.

Theorem 5.2. In the hypothesis of Theorem 4.1, $\mathcal{S} U_{C}(w, r, L)$ is a rational variety.

Proof. By hypothesis $d_{i}$ and $r$ are coprime, then the moduli space $\mathcal{S} U_{C_{i}}\left(r, L_{i}\right)$ is rational for any line bundle $L_{i} \in \operatorname{Pic}^{d_{i}}\left(C_{i}\right)$, see [KS99], [New75] and [New80]. Since $\mathscr{U}_{L}$ is a $\mathbb{P}^{r^{2}-1}$-bundle over the product $\mathcal{S} U_{C_{1}}\left(r, L_{1}\right) \times \mathcal{S} U_{C_{2}}\left(r, L_{2}\right)$, then it is a rational variety too. The assertion follows from corollary 5.1 .

\section{REFERENCES}

[Ati57] M. F. Atiyah, Vector bundles over an elliptic curve, Proc. London Math. Soc. (3) 7 (1957), 414-452. $\uparrow 4$

[BDS16] P. Barik, A. Dey, and B. N. Suhas, On the rationality of Nagaraj-Seshadri moduli space, Bull. Sci. Math. 140 (2016), no. 8, 990-1002, DOI 10.1016/j.bulsci.2016.06.001. $\uparrow 2$

[Bea13] A. Beauville, Theta Functions, Old and New, SMM 6 (2013). Open Problems and Surveys of Contemporary Mathematics. $\uparrow 1$

[BB12] M. Bolognesi and S. Brivio, Coherent systems and modular subavrieties of $\mathscr{S} \mathscr{U}_{C}(r)$, Internat. J. Math. 23 (2012), no. 4, 1250037, 23, DOI 10.1142/S0129167X12500371. $\uparrow 1$

[BB14] U. N. Bhosle and I. Biswas, Brauer group and birational type of moduli spaces of torsionfree sheaves on a nodal curve, Comm. Algebra 42 (2014), no. 4, 1769-1784, DOI 10.1080/00927872.2012.749263. $\uparrow 2$

[BF19a] S. Brivio and F. F. Favale, Genus 2 curves and generalized theta divisors, Bull. Sci. Math. 155 (2019), 112-140, DOI 10.1016/j.bulsci.2019.05.002. $\uparrow 1$

[BF19b] _ _ On Kernel Bundle over reducible curves with a node (2019). Preprint (2019). $\uparrow 2$

[Bri15] S. Brivio, A note on theta divisors of stable bundles, Rev. Mat. Iberoam. 31 (2015), no. 2, 601-608, DOI 10.4171/RMI/846. MR3375864 $\uparrow 1$

[Bri17] _ Families of vector bundles and linear systems of theta divisors, Internat. J. Math. 28 (2017), no. 6, 1750039, 16. $\uparrow 1$

[Bri18] _ Theta divisors and the geometry of tautological model, Collect. Math. 69 (2018), no. 1, 131-150. $\uparrow 1$

[BV12] S. Brivio and A. Verra, Plücker forms and the theta map, Amer. J. Math. 134 (2012), no. 5, 1247-1273, DOI 10.1353/ajm.2012.0034. MR2975235 $\uparrow 1$

[DS18] A. Dey and B. N. Suhas, Rationality of moduli space of torsion-free sheaves over reducible curve, J. Geom. Phys. 128 (2018), 87-98, DOI 10.1016/j.geomphys.2018.02.009. $\uparrow 2$ 
[Har77] R. Hartshorne, Algebraic geometry, Springer-Verlag, New York-Heidelberg, 1977. Graduate Texts in Mathematics, No. 52. $\uparrow 16$

[KS99] A. King and A. Schofield, Rationality of moduli of vector bundles on curves, Indag.Math.(NS) 10 (1999), no. $4,519-535 . \uparrow 1,16$

[Mum65] D. Mumford, Geometric invariant theory, Ergebnisse der Mathematik und ihrer Grenzgebiete, Neue Folge, Band 34, Springer-Verlag, Berlin-New York, 1965. $\uparrow 1$

[Ses67] C.S. Seshadri, Space of unitary vector bundles on a compact Riemann surface, Ann.of Math. 85 (1967), 303-336. $\uparrow 1$

[Ses82] _ Fibrés vectoriels sur les courbes algébriques, Conférences á l'E.N.S.-Juin 1980, rédigées par J.M. Drezet, Astérisque, vol. 96, Société Mathématique de France, 1982. $\uparrow 1$, 4, 5, 14

[New78] P.E. Newstead, Introduction to Moduli problems and orbit spaces, Lectures on Mathematics and Physics, Vol. 51, 1978. Tata Institute of fundamental Research, Bombay. $\uparrow 1$

[New80] P.E. Newstead, Correction to "Rationality of moduli spaces of stable bundles", Math. Ann. 249 (1980), 281-282. $\uparrow 16$

[New75] _ _ Rationality of moduli spaces of stable bundles, Math. Ann. 215 (1975), 251-268. $\uparrow 16$

[NMRS78] Narasimhan M.S. and Ramanan S., Geometry of Hecke Cycles-I, Springer, Berlin-New York, Vol. 8, 1978. Tata Inst. Fund. Res. Studies in Math. $\uparrow 9$

[Ram73] S. Ramanan, The moduli spaces of vector bundles over an algebraic curve, Math. Ann. 200 (1973), 69-84. $\uparrow 1,5$

[RTiB99] B. Russo and M. Teixidor i Bigas, On a conjecture of Lange, J. Algebraic Geom. 8 (1999), no. 3, $483-496$. $\uparrow 11$

[TiB11] M. Teixidor i Bigas, Vector bundles on reducible curves and applications, Grassmannians, moduli spaces and vector bundles, Clay Math. Proceedings 14 (2011), 169-180. $\uparrow 2,5,9$

[TiB95] _ Moduli spaces of Vector bundles on reducible curves, Amer.J. of Math. 117 (1995), no. 1, $125-139$. $\uparrow 2,5$

[Tu93] L. W. Tu, Semistable Bundles over an Elliptic Curve, Ad.in Math. 98 (1993), 1-26. $\uparrow 4$

Dipartimento di Matematica e Applicazioni, Università degli Studi di Milano-Bicocca, Via Roberto Cozzi, 55, I-20125 Milano, Italy

E-mail address: filippo.favale@unimib.it

Dipartimento di Matematica e Applicazioni, Università degli Studi di Milano-Bicocca, Via Roberto Cozzi, 55, I-20125 Milano, Italy

E-mail address: sonia.brivio@unimib.it 